\title{
Controversias sobre el rastreo de los niveles séricos de vitamina $D$ y su suplementación
}

Controversies about serum vitamin D levels screening and its supplementation

Vivian Alias D’Abate*, Chu Yoo* y Marcela Botargues*

\begin{abstract}
Resumen
Si bien los niveles bajos de vitamina $\mathrm{D}$ se han asociado con varios resultados de interés en salud, aún resulta motivo de controversia qué significa un nivel bajo, cual es la utilidad de su suplementación y cuales son sus potenciales efectos adversos.

En ese contexto, se realizó en el Servicio de Medicina Familiar y Comunitaria del Hospital Italiano un taller de discusión denominado "Actividad ECCO" (Evidencia Científica en la Clínica Cotidiana) en la que fueron presentados los resultados de estudios identificados que hubieran comparado el uso de vitamina $\mathrm{D}$ (con o sin suplementación de calcio) versus placebo, con el objetivo de discutir cuál es la evidencia actual para el rastreo de deficiencia de vitamina $D$ y para, eventualmente, recomendar o no su suplementación. Este artículo resume la evidencia identificada y las conclusiones consensuadas en dicha actividad.

\section{Abstract}

Although low levels of vitamin D have been associated with several health outcomes, it is controversial what a low level means, the usefulness of its supplementation and its potential adverse effects.

In this context, a workshop called "ECCO Activity" (Scientific Evidence in the Daily Clinic) was held in the Family and Community Medicine Division of Hospital Italiano de Buenos Aires, where the results of identified studies that compared the use of vitamin D (with or without calcium supplementation) versus placebo, with the aim of discussing what is the current evidence for screening of vitamin D deficiency and to, eventually, recommend or not its supplementation. This article summarizes the identified evidence and the agreed conclusions in that activity.
\end{abstract}

Palabras clave: vitamina D, deficiencia, rastreo, tratamiento. Key words: vitamin D, deficiency, tracking, treatment.

Alias D’Abate V, Yoo C y Botargues M. Controversias sobre el rastreo de los niveles séricos de vitamina D y su suplementación. Evid Act Pract Ambul. 2017; 20(4): 102-104.

Rastreo de la deficiencia de vitamina $D$ en la población general Teniendo en cuenta que entre el 50 y el $80 \%$ de la población general tiene niveles bajos de vitamina $\mathrm{D}$ y que pocos alimentos la contienen, las sociedades de especialistas en endocrinología y ginecología de Norteamérica y de Argentina, han comenzado a recomendar su dosaje en la población con osteoporosis ${ }^{1,2,3,4,5}$. Sin embargo, la fundamentación de estas recomendaciones proviene de estudios observacionales y entraña los riesgos de sacar conclusiones de poblaciones pequeñas y poco representativas de la población general (más parecidas a las que atienden los especialistas), trasladando sus recomendaciones al tratamiento y a la consejería de la población que atendemos en atención primaria.

Por otra parte no hay un total acuerdo sobre los valores de vitamina D considerados "óptimos"6. Algunas sociedades médicas como el Instituto of Medicina de Estados Unidos (en inglés IOM) proponen como valor óptimo al rango comprendido entre 20 y 40 $\mathrm{ng} / \mathrm{mL}$ (50 a $100 \mathrm{nmol} / \mathrm{L}$ ), mientras que otros expertos proponen valores entre 30 and $50 \mathrm{ng} / \mathrm{mL}$ (75 a $125 \mathrm{nmol} / \mathrm{L}$ ). Se considera un nivel "deficiente" a valores inferiores $10 \mathrm{ng} / \mathrm{mL}$, ya que la salud del esqueleto podría comprometerse por menor absorción de calcio a nivel intestinal. No obstante, hay que tener en cuenta que estas consideraciones son en base a observaciones realizadas en poblaciones con patología de absorción, desnutrición severa, osteomalacia, etc. y no en la población general. Además, existen valores de zonas grises (entre 10 y $30 \mathrm{ng} / \mathrm{mL}$ ), cuyo significado para la salud no queda claro actualmente. Por otro lado, el dosaje de vitamina $D$ se realiza por diferentes métodos, cada uno con sus características operativas y sin que su medición esté estandarizada ${ }^{5,6,7}$.

Actualmente, la Fuerza de Tareas para los Servicios Preventivos de los Estados Unidos (USPSTF, su sigla en inglés) concluye que la evidencia actual es insuficiente para evaluar el equilibrio entre los beneficios y los daños del rastreo de déficit de vitamina $\mathrm{D}$ en adultos asintomáticos (Recomendación $\mathrm{I}^{8}$.

Por otro lado, algunos expertos recomiendan rastrear la deficiencia de vitamina $D$ en los individuos con mayor riesgo de presentar deficiencia mediante alguna prueba confiable $e^{1,2,3,4}$. Las entidades clínicas que aumentan ese riesgo son:

- Las enfermedades asociadas con malabsorción: celiaquía, enfermedad inflamatoria intestinal, insuficiencia pancreática o by pass gástrico.

- La enfermedad hepática y la enfermedad renal crónica.

- Los fármacos: fenitoína, fenobarbital, antirretrovirales, corticoides.

- La falta de exposición al sol por alguno de los siguientes motivos: institucionalización en un lugar cerrado sin jardín, piel cubierta por prendas de vestir (no aplicable en el Área Metropolitana de Buenos Aires ya que nuestro clima es cada vez más tropical), y uso estricto de protección solar.

\section{Utilidad del tratamiento con vitamina D}

La suplementación con vitamina $D$ no ha demostrado disminución de la incidencia de fracturas ni de la mortalidad global ${ }^{9,10}$. Sin embargo, sí se ha observado una disminución significativa de la incidencia de fractura de cadera en población anciana frágil o institucionalizada y también una disminución de la incidencia de caídas en esta población ${ }^{10}$. Es de destacar que ha sido documentada una reducción de más del $30 \%$ de la frecuencia de caídas en población anciana institucionalizada (RR 0,66; IC 95\% $0,50$ a 0,88$)$, por lo que en esta población podría no ser necesario el dosaje y sí el tratamiento.

\section{Vitamina $D$ y riesgo de fractura}

Reanálisis de los datos del ensayo clínico "Iniciativa de salud de las mujeres, calcio/vitamina D" ("Women Health Initiative /CaD study")

EI WHI/CaD ${ }^{11}$ fue un ensayo clínico aleatorizado de siete años de seguimiento que había incluido a 36.282 mujeres de la comunidad de 50 a 79 años de edad y las había asignado a dos ramas de tratamiento: 1) $1.000 \mathrm{mg}$ de calcio más $400 \mathrm{UI}$ de vitamina $\mathrm{D}$; 2) placebo.

El tratamiento con calcio y vitamina $D$ mostró un efecto pequeño en el aumento de la densidad mineral ósea $(1,06 \%$ en ca-dera $)^{1}$, sin efecto sobre la disminución de la incidencia global de

* Servicio de Medicina Familiar y Comunitaria del Hospital Italiano de Buenos Aires. vivian.alias@ hospitalitaliano.org.ar 
fracturas (HR 0,88; 0,72 a 1,08). Solo se observó una reducción significativa de la incidencia fractura de cadera (HR 0,71; 0,52 a 0,97 ) en el análisis restringido a los pacientes adherentes al tratamiento y el riesgo de litiasis renal fue mayor en el subgrupo que recibió suplementación de calcio y vitamina D (HR 1,17; 1,02 a 1,34).

RECORD study (Randomised Evaluation of Calcium Or vitamin D) Incluyó 5.292 personas mayores de 70 años (85\% mujeres), con antecedentes de fracturas asociadas a traumatismos menores, que fueron asignadas en forma factorial a uno de los siguientes cuatro grupos: 1) vitamina D $800 \mathrm{UI}$; 2) calcio $1.000 \mathrm{mg} / \mathrm{día}$; 3) calcio más vitamina $D ;$ o 4) placebo. El seguimiento luego de la intervención fue de dos a cinco años y el análisis fue por intención de tratar. El tratamiento con vitamina $D$ o vitamina $D$ y calcio no mostró reducción de la incidencia de fracturas ${ }^{12}$.

\section{Otros estudios}

Un meta-análisis de cinco ECCA que habían comparado la suplementación de vitamina $\mathrm{D}$ (400 a $1.370 \mathrm{UI}$ diarias) con placebo en más de 14.000 participantes reportó que su suplementación en ausencia de suplementación con calcio no redujo la incidencia de fractura (RR 1,03; 0,84 a 1,26$)^{13}$

En relación a los efectos de la vitamina $D$ sobre el riesgo de fractura, en comparación al calcio, los estudios mostraron resultados mixtos debido a las diferencias de las poblaciones y al diseño de los estudios.

\section{Vitamina $D$ y riesgo de caídas}

Un meta-análisis de cinco $\mathrm{ECCA}^{10}$ evaluó el riesgo de sufrir al menos una caída en un grupo de pacientes tratados con 800 $\mathrm{UI} / \mathrm{d}$ de vitamina $\mathrm{D}$ (con o sin calcio) durante dos a 36 meses, comparando los resultados de esta intervención con un grupo control (un total de 1.677 personas). Estos ensayos clínicos habían incluido una población de pacientes frágiles: mayores de 70 años, personas institucionalizadas, pacientes con dificultades en la movilidad o con múltiples co-morbilidades; y en dos estudios en particular, pacientes con antecedentes personales de caídas. Cuando se realizó un análisis global, la rama asignada a vitamina $D$ evidenció una incidencia menor de caídas, aunque estadísticamente no significativa $(0,84 ; 0,69$ a 1,02$)$. Sin embargo, y dado que los estudios tenían una alta heterogeneidad, luego de excluir los dos estudios en los que también había habido una co-intervención con calcio, la administración exclusiva de vitamina $D$ (tres ensayos clínicos de altísima homogeneidad) se asoció con una disminución de al menos una caída $(0,65 ; 0,52$ a 0,81 ) de una densidad de incidencia de 58,6 caídas por cada 100 personas año de seguimiento en el grupo control a 54 en el grupo intervención.

Otro meta-análisis de cinco $\mathrm{ECCA}^{10}$, que incluyó un total de 1.399 individuos, comparó la efectividad del tratamiento con vitamina $D$ con 400 a $1.000 \mathrm{lU} / \mathrm{d}$ (con o sin calcio) contra un grupo control, usando como punto final el número de caídas por persona. Los asignados a la rama vitamina $D$ tuvieron significativamente un tercio menos de caídas $(0,66 ; 0,50$ a 0,88).

El tratamiento con vitamina $D$ se asoció con una reducción significativa del número de caídas por persona ${ }^{10,11,12,13}$. Sin embargo, su resultados fueron influenciados por un $\mathrm{ECCA}^{13}$ realizado en una población de ancianos institucionalizados frágiles, con una razón de densidad de incidencia de 0,66 (IC $95 \%$ 0,50 a 0,88).

\section{Vitamina $D$ y mortalidad}

Si bien la mortalidad no fue objetivo primario de ninguno de los estudios mencionados, esta disminuyó en los participantes asignados a tratamiento con vitamina $D$ con o sin calcio $(0,83 ; 0,70$ a 0,99), fundamentalmente a costa de la reducción del riesgo en ancianos institucionalizados $(0,72 ; 0,56 \text { a } 0,94)^{10}$.

Se prevé la publicación de nuevos estudios que evalúen el efecto de la vitamina $D$ sobre enfermedades oncológicas, fracturas y mortalidad global .

\section{Consenso luego de la discusión}

En el proceso de decisiones compartidas recomendamos explicarle a el o la paciente lo mencionado anteriormente y los potenciales riesgos de aumento de la incidencia de litiasis renal $(1,17$; $1,02$ a 1,34$)^{14}$, hipercalcemia leve (especialmente con calcitriol), síntomas gastrointestinales (especialmente con vitamina $D+$ calcio) $)^{5,15,14}$ y un aumento de $30 \%$ del riesgo cardiovascular de acuerdo a lo comunicado por cohortes prospectivas, sin que contemos con ECCA que hayan evaluado esta asociación.

Respecto del riesgo de toxicidad de la vitamina $D$, nos parece mínimo ya que se observa con dosis mucho más altas $(4.000$ UI diarias) que las usualmente empleadas en nuestra práctica clínica.

De resultar el proceso de decisión a favor del tratamiento recomendamos el uso de vitamina D3 por sobre la D2 y el calcitriol, por su mayor duración y por su capacidad de incrementar los niveles de $25 \mathrm{HO}$ colecalciferol.

Respecto de la dosis, un meta-análisis de estudios aleatorizados no demostró diferencia sobre la reducción de riesgo de fractura en relación a las dosis de vitamina $D$ utilizada ${ }^{5,10,16}$. Por otro lado, no existe una relación lineal entre la dosis de suplementación y el nivel sérico de $25 \mathrm{OH} \mathrm{D}$, pero en individuos con un nivel basal bajo $(<10 \mathrm{ng} / \mathrm{mL})$ puede esperarse un incremento de 1 a $1,5 \mathrm{ng} / \mathrm{mL}$ por cada $100 \mathrm{UI}$ de $25 \mathrm{OH} \mathrm{D}$ recibidos y en individuos con niveles basales mayores a $20 \mathrm{ng} / \mathrm{mL}$, un incremento menor (0,5ng/mL por cada $100 \mathrm{UI}$ recibidos).

Cuando se administran los suplementos diariamente, las vitaminas D2 y D3 tienen una potencia equivalente. Si dicha administración es intermitente (mensual, bimestral o trimestral), como la vitamina D3 tiene mayor vida media que la vitamina D2, se recomienda su elección ${ }^{3}$.

No recomendamos la administración de mega dosis anual de 500.000 UI de vitamina D3 ya que se asoció con un incremento del riesgo de caídas y fracturas en ancianos.

\section{Conclusiones finales}

No está recomendado el rastreo universal de hipovitaminosis D en la población general y se desconoce su verdadera utilidad en las personas con osteoporosis que no tienen factores de riesgo para la deficiencia de vitamina $\mathrm{D}$. El aporte o suplemento terapéutico de vitamina $D$ con o sin calcio no tiene efecto sobre la disminución de la incidencia de fracturas, ni en la población sana ni en población con osteoporosis, como tampoco en población con osteoporosis y fracturas ya establecidas. Sin embargo la suplementación en pacientes ancianos frágiles o institucionalizados podría aportar beneficios en cuanto a la reducción del riesgo de caídas.

Recibido en 01/06/16 y aceptado el 30/04/17 
Referencias bibliográficas

1. Holick M y col. Evaluation, Treatment, and Prevention of Vitamin D Deficiency: an Endocrine Society Clinical Practice Guideline. J Clin Endocrinol Metab. 2011; 96(7):19111930 .

2. Dawson-Hugues B y col. IOF position statement: vitamin D recommendations for older adults. IOF position statement: vitamin $\mathrm{D}$ recommendations for older adults. Osteoporos.

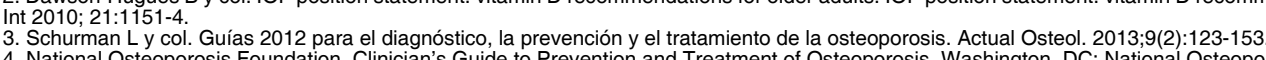

4. National Osteoporosis Foundation. Clinician's Guide to Prevention and Treatment of Osteoporosis. Washington, DC: National Osteoporosis Foundation; 2014

5. Dawson-Hughes B. Vitamin D deficiency in adults: Definition, clinical manifestations and treatment. UpToDate. Waltham, MA: UpToDate Inc. Disponible en URL:http://www.

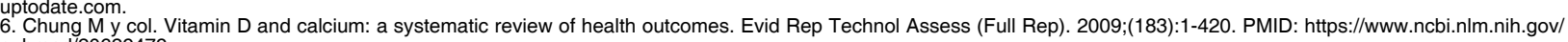

7. LeFevre ML. Screening for Vitamin D Deficiency in Adults: U.S. Preventive Services Task Force Recommendation Statement. Ann Intern Med. 2015;162(2):133. PMID: https://

8. LeBlanc E y col. Screening for Vitamin D Deficiency: A Systematic Review for the U.S. Preventive Services Task Force. Ann Intern Med. 2015;162:109-122. doi:10.7326/ M14-1659.

9. Avenell A y col. Vitamin D and vitamin D analogues for preventing fractures in post-menopausal women and older men. Cochrane database Syst Rev. $2014 ; 4(4)$ :CD000227. PMID: https://www ncbi.nlm nih gov/pubmed/24729336.

10. Bjelakovic G y col. Vitamin D supplementation for prevention of mortality in adults. Cochrane Database Syst Rev. 2011;(7):CD007470. doi: 10.1002/14651858.CD007470. pub2.

11. Bolland M y col. Calcium and vitamin D supplements and health outcomes: a reanalysis of the Women's Health Initiative (WHI) limited-access data set. Am J Clin Nutr. 2011;94(4):1144-9. doi: 10.3945/ajcn.111.015032. Epub 2011 Aug 31.

12. Grant A y col. Oral vitamin D3 and calcium for secondary prevention of low-trauma fractures in elderly people (Randomised Evaluation of Calcium Or vitamin D, RECORD): a randomized placebo-controlled trial. Lancet 2005; 365:1621-28.

13. Chung $\mathrm{M}$ y col. Vitamin $\mathrm{D}$ with or without calcium supplementation for prevention of cancer and fractures: an updated meta-analysis for the U.S. Preventive Services Task Force. Ann Intern Med. 2011;155(12):827-38. doi: 10.7326/0003-4819-155-12-201112200-00005.

14. Meyer H. Should vitamin D supplements be recommended to prevent chronic diseases? BMJ 2015;350:h321 doi: $10.1136 / \mathrm{bmj}$.h321

15. Rosen $\mathrm{H}$ y col. Calcium and vitamin D supplementation in osteoporosis. UpToDate. Waltham, MA: UpToDate Inc. Disponible en URL:http://www.uptodate.com.

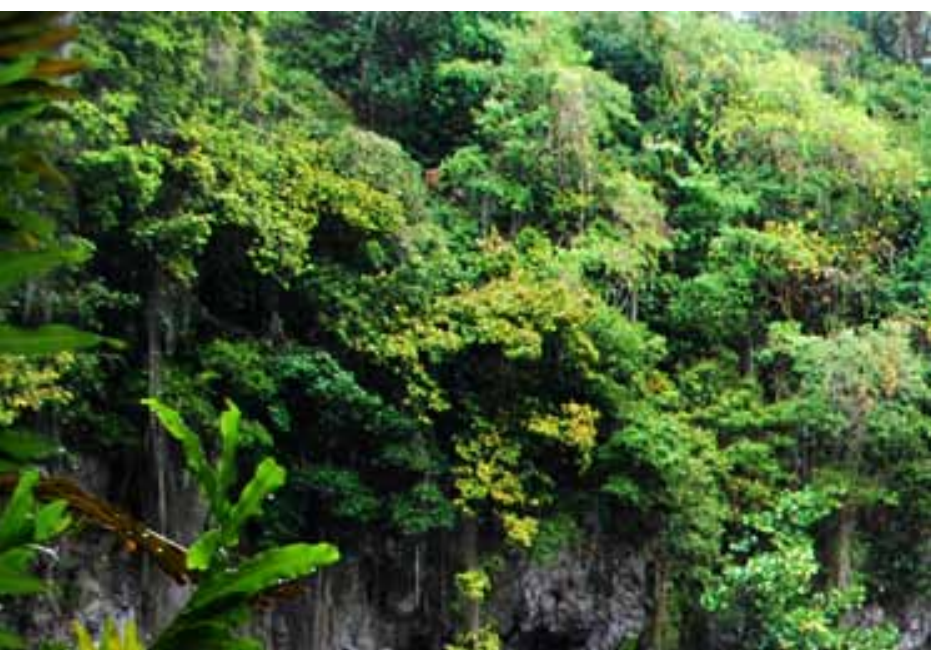

Cortesía de Silvia Spina 